\title{
Energy harvesting in a delayed and excited Duffing harvester device
}

\author{
Z. Ghouli ${ }^{1}$, M. Hamdi ${ }^{2}$, F. Lakrad ${ }^{1}$, M. Belhaq ${ }^{1}$ \\ ${ }^{1}$ Laboratory of Renewable Energy and Structural Dynamics, University Hassan II-Casablanca, Morocco \\ ${ }^{2}$ University Mohammed I Oujda, FST-Al Hoceima, Morocco
}

\begin{abstract}
We explore quasi-periodic (QP) vibration-based energy harvesting (EH) in a forced nonlinear oscillator under time delay. The energy harvesting system consists in a delayed Duffing oscillator subject to harmonic excitation coupled to an electric circuit through an electromechanical coupling mechanism. We consider the case of primary resonance for which the frequency of the excitation is near the natural frequency of the oscillator. Application of the double-step perturbation method enables the approximation of the amplitude of the QP vibrations used to extract the average powers from the harvester device. Results shown that for a small value of delay amplitude, the periodic vibration-based EH can be extracted in a narrow range near the resonance, while QP vibration-based EH can be extracted over broad ranges of excitation. For relatively increasing value of the delay amplitude, only QP vibration-based EH can be extracted.
\end{abstract}

\section{Introduction}

To improve the EH capability, the limitation of the linear attachment has been overcome by using nonlinearities, as for example, a cubic nonlinear component. This essential nonlinearity extends the bandwidth of the harvester over broad range of excitation frequencies, either in the case where the harvesting system is designed as monostable with hardening characteristic [1] or in the case of bistablity; see for instance [2] and references therein. However, the nonlinear attachment is no longer ensured, especially when the response suffers jump phenomena near the resonance[3].

To overcome this problem, the idea of using QP vibrations is proposed. Indeed, it was reported recently that introducing a modulated delay amplitude in a pure selfexcited oscillator coupled to an electromagnetic energy harvesting system produces large amplitude QP vibrations performing in broader range of parameters $[4,5]$.

The purpose of this work is to enhance the energy harvesting performance in a harvester system with Duffingtype nonlinear attachment and subject to harmonic excitation using time delay. The energy harvester system consists in a delayed lumped-parameter Duffing oscillator coupled to an electrical circuit through an electromagnetic coupling mechanism. Special attention is paid to the effect of different delay and coupling parameters on the energy harvesting performance taking advantage of large-amplitude QP vibrations induced by time delay.

in Section 2 we present the electromagnetic VEH system and derive the periodic response as well as the harvested power near the primary resonance using the multiple scales method. Section 3 is devoted to the investigation of the QP response and the QP vibration-based EH. The conclusion is given in the last Section.

\section{Model description and periodic energy harvesting}

Consider an energy harvester device under time delay in the position such that the governing system of equations can be written in the dimensionless form as

$$
\begin{aligned}
\ddot{x}(t)+\delta \dot{x}(t)+\omega_{0}^{2} x(t)+\gamma x(t)^{3}-\chi v(t) & =\alpha x(t-\tau)+f \cos (\lambda t) \\
\dot{v}(t)+\beta v(t)+\kappa \dot{x}(t) & =0
\end{aligned}
$$

where $x(t)$ is the relative displacement of the rigid mass $\mathrm{m}, v(t)$ is the voltage across the load resistance, $\delta$ is the mechanical damping ratio, $\gamma$ is the stiffness parameter, $\chi$ is the piezoelectric coupling term in the mechanical equation, $\kappa$ is the piezoelectric coupling term in the electrical circuit equation, $\beta$ is the reciprocal of the time constant of the electrical circuit, $\alpha$ and $\tau$ are, respectively, the gain and time delay, while $f$ and $\lambda$ are, respectively, the amplitude and the frequency of the excitation.

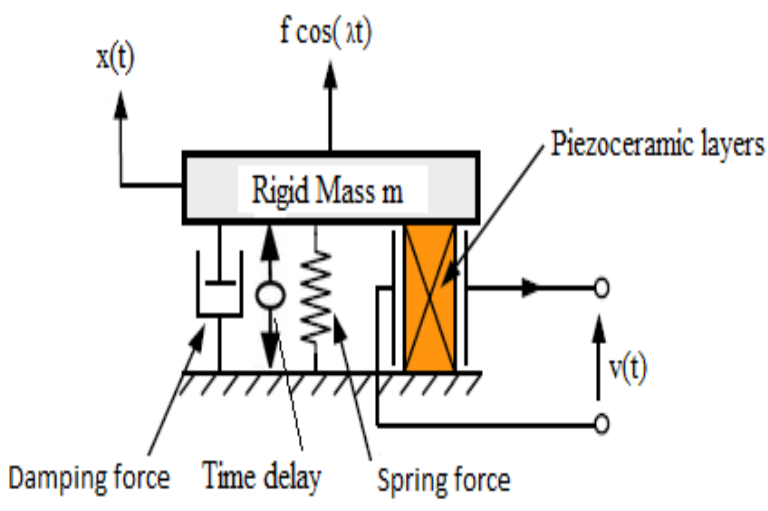

Fig. 1. Schematic of the energy harvesting system 
To study the response of the system near the primary resonance, we introduce the resonance condition $\lambda=\omega_{0}+$ $\sigma$ where $\sigma$ is a detuning parameter and we use the multiple scales technique [6] by introducing a bookkeeping parameter $\epsilon$ such that parameters can be scaled as $\delta=\epsilon \tilde{\delta}, \gamma=$ $\epsilon \tilde{\gamma}, \chi=\epsilon \tilde{\chi}, \alpha=\epsilon \tilde{\alpha}, f=\epsilon \tilde{f}, \sigma=\epsilon^{2} \tilde{\sigma}$. Thus, Eqs. (1) and (2) take the form

$$
\begin{gathered}
\ddot{x}(t)+\omega_{0}^{2} x=\epsilon\left[-\tilde{\delta} \dot{x}(t)-\tilde{\gamma} x(t)^{3}+\tilde{\chi} v(t)+\tilde{\alpha} x(t-\tau)+\tilde{f} \cos (\lambda t)\right] \\
\dot{v}(t)+\beta v(t)+\kappa \dot{x}(t)=0
\end{gathered}
$$

Applying the multiple scale technique, a solution to Eqs. (3) and (4) can be sought in the forme

$$
\begin{aligned}
& x(t)=x_{0}\left(T_{0}, T_{1}, T_{2}\right)+\epsilon x_{1}\left(T_{0}, T_{1}, T_{2}\right)+\epsilon^{2} x_{2}\left(T_{0}, T_{1}, T_{2}\right)+O\left(\epsilon^{3}\right) \\
& v(t)=v_{0}\left(T_{0}, T_{1}, T_{2}\right)+\epsilon v_{1}\left(T_{0}, T_{1}, T_{2}\right)+\epsilon^{2} v_{2}\left(T_{0}, T_{1}, T_{2}\right)+O\left(\epsilon^{3}\right)
\end{aligned}
$$

where $T_{0}=t, T_{1}=\epsilon t$ and $T_{2}=\epsilon^{2} t$. Substituting (5) and (6) into (3) and (4), extracting systems of equation at different order of $\epsilon$ and eliminating secular terms as usual, we obtain up to the first order the modulation equations

$$
\left\{\begin{array}{l}
\frac{d a}{d t}=\frac{C_{1}}{2 \omega_{0}} a+\frac{f}{2 \omega_{0}} \sin (\varphi) \\
a \frac{d \varphi}{d t}=\frac{C_{2}}{2 \omega_{0}} a-\frac{C_{3}}{2 \omega_{0}} a^{3}+\frac{f}{2 \omega_{0}} \cos (\varphi)
\end{array}\right.
$$

where $a$ and $\theta$ are the amplitude and the phase, and $C_{1}=$ $-\delta \omega_{0}-\alpha \sin \left(\omega_{0} \tau\right)-\frac{\kappa \beta \chi \omega_{0}}{\left(\beta^{2}+\omega_{0}^{2}\right)}, C_{2}=2 \omega_{0} \sigma+\alpha \cos \left(\omega_{0} \tau\right)-$ $\frac{\kappa \chi \omega_{0}^{2}}{\left(\beta^{2}+\omega_{0}^{2}\right)}, C_{3}=\frac{3 \gamma}{4}$ and $\varphi=\tilde{\sigma} T_{2}-\theta$.

The solution to the first order can be written as $x_{0}\left(T_{0}, T_{1}, T_{2}\right)=$ $a \cos \left(\omega_{0} t+\theta\right)$ and $v_{0}\left(T_{0}, T_{1}, T_{2}\right)=V \cos \left(\omega_{0} t+\theta+\arctan \frac{\beta}{\omega_{0}}\right)$ and the relation between the voltage amplitude $V$ and the vibration amplitude $a$ is given by

$$
V=\frac{\kappa \omega_{0}}{\sqrt{\beta^{2}+\omega_{0}^{2}}} a
$$

The steady state response of system (7) are determined by setting $\frac{d a}{d t}=\frac{d \varphi}{d t}=0$. We obtain the following sixth-order algebraic equation in $a$

$$
C_{3}^{2} a^{6}-2 C_{2} C_{3} a^{4}+\left(C_{1}^{2}+C_{2}^{2}\right) a^{2}-f^{2}=0
$$

An expression for the average power is obtained by integrating the dimensionless form of the instantaneous power $P(t)=\beta v(t)^{2}$ over the period of the excitation $T$. This is given by

$$
P_{a v}=\frac{1}{T} \int_{0}^{T} \beta v^{2} d t
$$

where $T=\frac{2 \pi}{\lambda}$. Then, the average power reads

$$
P_{a v}=\frac{1}{2}\left(\frac{\beta \kappa^{2} \omega_{0}^{2}}{\beta^{2}+\omega_{0}^{2}}\right) a^{2}
$$

where $a$ is obtained from Eq. (9).

\section{Quasi-periodic energy harvesting}

In this Section, we investigate the QP response as well as the QP vibration-based energy harvesting using the secondstep perturbation method [7]. Up to the second order the modulation equations are given by

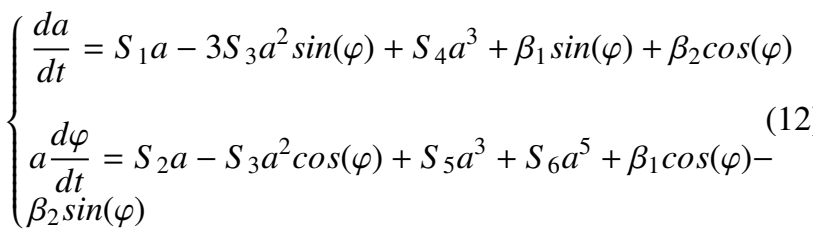

where $S_{1}, S_{2}, S_{3}, S_{4}, S_{5}, S_{6}, \beta_{1}$, and $\beta_{2}$ are given in Appendix. We transform the polar form (12) to the following Cartesian system using the variable change $u=a \cos \varphi$ and $w=-a \sin \varphi$

$$
\left\{\begin{array}{l}
\frac{d u}{d t}=S_{2} w+\beta_{2}+\eta\left\{S_{1} u+2 S_{3} u w+\left(S_{4} u+S_{5} w\right)\left(u^{2}+w^{2}\right)+\right. \\
\left.S_{6}\left(u^{2}+w^{2}\right)^{2} w\right\} \\
\frac{d w}{d t}=-S_{2} u-\beta_{1}+\eta\left\{S_{1} w+S_{3} w^{2}+S_{3}\left(u^{2}+w^{2}\right)+\right. \\
\left.\left(S_{4} u-S_{5} w\right)\left(u^{2}+w^{2}\right)-S_{6}\left(u^{2}+w^{2}\right)^{2} w\right\}
\end{array}\right.
$$

Applying the method of multiple scales on the slow flow (13), one obtains the following autonomous slow-slow flow system

$$
\left\{\begin{array}{l}
\frac{d R}{d t}=S_{4} R^{3}+\left(S_{1}-\alpha_{2} S_{3}+2 \alpha_{1}^{2} S_{4}+2 \alpha_{2}^{2} S_{4}\right) R \\
R \frac{d \psi}{d t}=S_{6} R^{5}+\left(S_{5}+6\left(\alpha_{1}^{2}+\alpha_{2}^{2}\right) S_{6}\right) R^{3}+ \\
\left(\left(2 \alpha_{1}^{2}+2 \alpha_{2}^{2}\right) S_{5}+\left(3 \alpha_{1}^{4}+3 \alpha_{2}^{4}+6 \alpha_{1}^{2} \alpha_{2}^{2}\right) S_{6}\right) R
\end{array}\right.
$$

where $\alpha_{1}=\frac{\beta_{1}}{S_{2}}, \alpha_{2}=\frac{\beta_{2}}{S_{2}}$ and $R$ and $\psi$ are, respectively, the amplitude and the phase of the slow flow limit cycle.

Equilibria of this slow-slow flow system (14) determine the QP solutions of the original system (1),(2). The nontrivial equilibrium is obtained by setting $\frac{d R}{d t}=0$ and given by

$$
R=\sqrt{\frac{-S_{1}+\alpha_{2} S_{3}-2 \alpha_{1}^{2} S_{4}-2 \alpha_{2}^{2} S_{4}}{S_{4}}}
$$

Thus, the approximate periodic solution of the slow flow (13) is given by

$$
\begin{gathered}
u(t)=R \cos (\varphi t)-\alpha_{1} \\
w(t)=-R \sin (\varphi t)-\alpha_{2}
\end{gathered}
$$

The amplitude $a(t)$ of the QP oscillations reads

$$
a(t)=\sqrt{\left[R^{2}+\alpha_{1}^{2}+\alpha_{2}^{2}\right]-\left[2 \alpha_{1} R \cos (\varphi t)-2 \alpha_{2} R \sin (\varphi t)\right]}
$$

and the envelope of the QP modulation is delimited by $a_{\text {min }}$ and $a_{\max }$ given by

$$
a_{\text {min }}=\min \left\{\sqrt{\left[R^{2}+\alpha_{1}^{2}+\alpha_{2}^{2}\right] \pm\left[2 \alpha_{1} R \pm 2 \alpha_{2} R\right]}\right\}
$$




$$
a_{\text {max }}=\max \left\{\sqrt{\left[R^{2}+\alpha_{1}^{2}+\alpha_{2}^{2}\right] \pm\left[2 \alpha_{1} R \pm 2 \alpha_{2} R\right]}\right\}
$$

The average QP power can be written as

$$
P_{a v Q P}=\frac{1}{2}\left(\frac{\beta \kappa^{2} v^{2}}{\beta^{2}+v^{2}}\right) a^{2}
$$

where now $a$ is derived from Eqs. (19) and (20) and $v$ represents the frequency of slow flow limit cycle corresponding to the frequency of the QP modulation.

Figures 2 and 3 show, respectively, the variation of the amplitude of the periodic and QP responses and the output average power amplitude $\left(P_{a v}, P_{a v Q P}\right)$ versus the external frequency $\lambda$ for fixed values of parameters and for $\alpha=0.2$ (Fig. (2)) and $\alpha=0.4$ (Fig. (3)). The periodic response is given by (9) and the QP modulation envelope is obtained from Eqs. (19) and (20). Also, the average power for the periodic response is given by Eq. (11), while that for the QP response is deduced from (21). The solid lines represent analytical approximations, dashed lines correspond to unstable solution, while circles indicate numerical simulations. Inset in the figures are shown time histories of the amplitudes and the average power responses. Figure 2 shows that for a small value of delay amplitude $\alpha=0.2$, the periodic vibration-based energy harvesting can be extracted in a narrow range near the resonance (solid line in the power response), thereby limiting the bandwidth of the harvester. Instead, QP vibration-based energy harvesting can be extracted over broad range of excitation frequencies far from the resonance, as depicted inset in Fig. $2 b$.

Figure 3 indicates that for relatively increased value of the delay amplitude, $\alpha=0.4$, periodic vibrations turn to unstable, whereas the QP-based energy harvesting can be extracted away from the resonance with improved performance, as shown inset in Fig. $3 b$ by time histories of the average power responses.

\section{Conclusion}

The EH performance in a delayed forced Duffing oscillator coupled to an piezoelectric harvesting device is examined near the primary resonance in the case of a mono-stable oscillator. The method of multiple scales is performed twice to obtain the slow-slow flows of the EH system. The periodic and QP solutions as well as the corresponding power amplitudes are obtained and the influence of different system parameters on the EH performance is reported. It was shown that for a small value of delay amplitude, the periodic vibration-based $\mathrm{EH}$ can be extracted in a narrow range near the resonance, while QP vibration-based $\mathrm{EH}$ can be extracted over broad ranges of excitation away from the resonance. For relatively increasing value of the delay amplitude, EH can be extracted only from QP vibrations over broad bands of excitation frequencies.

\section{Appendix}

$$
\begin{array}{r}
S_{1}=-\frac{\delta}{2}-\frac{1}{2} \alpha \sin (\tau)-\frac{1}{4} \alpha^{2} \cos (\tau) \sin (\tau)-\frac{\chi \kappa \beta}{2\left(\beta^{2}+\omega_{0}^{2}\right)} \\
-\frac{\chi \kappa \alpha\left(\beta \cos (\tau)-\omega_{0} \sin (\tau)\right)}{8\left(\beta^{2}+\omega_{0}^{2}\right)}+\frac{\chi^{2} \kappa^{2} \beta \omega_{0}}{4\left[\left(\beta^{2}-\omega_{0}^{2}\right)^{2}+4 \beta^{2} \omega_{0}^{2}\right]}
\end{array}
$$
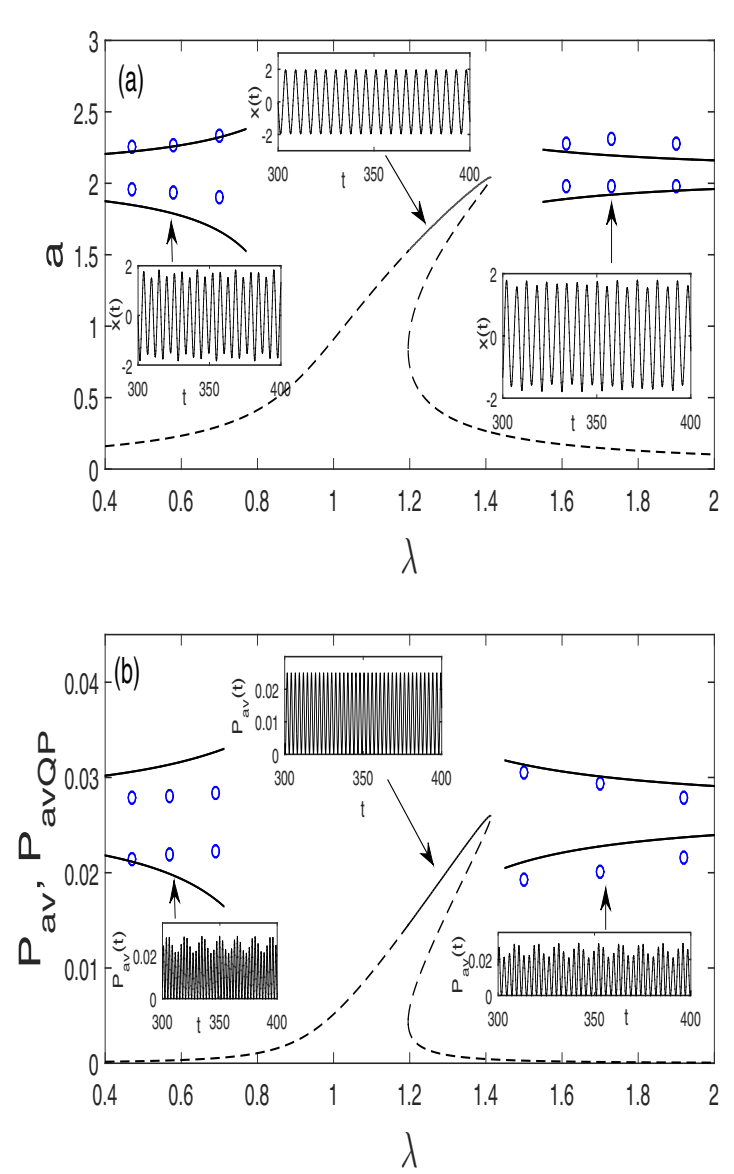

Fig. 2. Vibration and powers amplitudes vs $\lambda$ for $f=0.2, \chi=$ $0.05, \kappa=0.5$ and $\alpha=0.2$. Analytical prediction (solid lines for stable and dashed line for unstable) and numerical simulation (circles).

$$
\begin{gathered}
S_{2}=\lambda-\omega_{0}+\frac{\delta^{2}}{8}+\frac{1}{2} \alpha \cos (\tau)+\frac{1}{8} \alpha^{2} \cos ^{2}(\tau)-\frac{1}{8} \alpha^{2} \sin ^{2}(\tau) \\
-\frac{\chi \kappa \omega_{0}}{2\left(\beta^{2}+\omega_{0}^{2}\right)}-\frac{\chi \kappa \alpha\left(\beta \sin (\tau)+\omega_{0} \cos (\tau)\right)}{8\left(\beta^{2}+\omega_{0}^{2}\right)}-\frac{\chi^{2} \kappa^{2}\left(\beta^{2}-\omega_{0}^{2}\right)}{4\left[\left(\beta^{2}-\omega_{0}^{2}\right)^{2}+4 \beta^{2} \omega_{0}^{2}\right]} \\
S_{3}=\frac{3}{8} \gamma f \\
S_{4}=\frac{3}{16} \gamma \delta+\frac{3}{8} \alpha \gamma \sin (\tau)+\frac{3 \gamma \kappa \beta}{8\left(\beta^{2}+\omega_{0}^{2}\right)}+\frac{3 \gamma \kappa \beta \chi}{16\left(\beta^{2}+\omega_{0}^{2}\right)} \\
S_{5}=-\frac{3}{8} \gamma-\frac{3}{16} \alpha \gamma \cos (\tau)+\frac{3 \gamma \kappa \omega_{0}}{8\left(\beta^{2}+\omega_{0}^{2}\right)}-\frac{3 \gamma \kappa \omega_{0}}{16\left(\beta^{2}+\omega_{0}^{2}\right)} \\
S_{6}=\frac{15}{256} \gamma^{2}
\end{gathered}
$$$$
\beta_{1}=\frac{1}{2} f+\frac{1}{8} \alpha f \cos (\tau)-\frac{f \chi \kappa \omega_{0}}{8\left(\beta^{2}+\omega_{0}^{2}\right)}
$$$$
\beta_{2}=\frac{1}{8} \delta f-\frac{1}{8} \alpha f \sin (\tau)-\frac{f \chi \kappa \beta}{8\left(\beta^{2}+\omega_{0}^{2}\right)}
$$ 

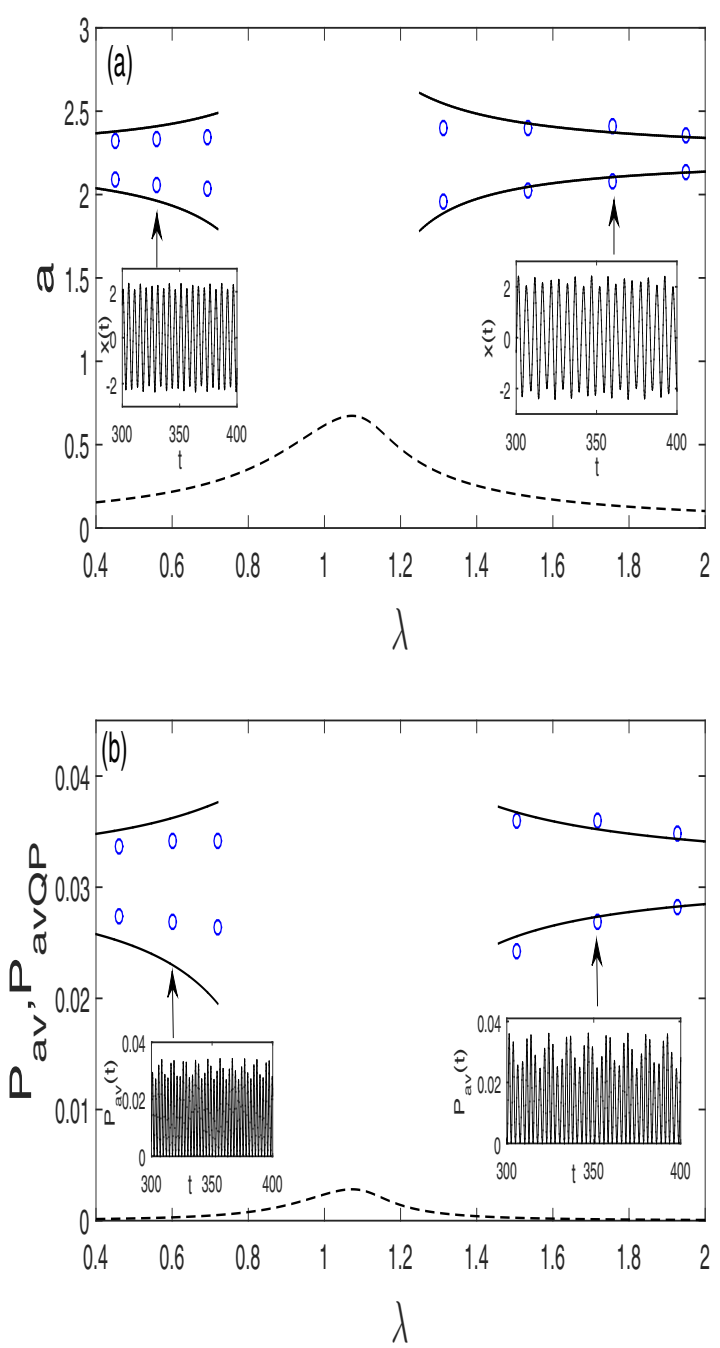

Fig. 3. Vibration and power amplitudes vs $\lambda$ for $f=0.2, \chi=$ $0.05, \kappa=0.5$ and $\alpha=0.4$. Analytical prediction (solid lines for stable and dashed line for unstable) and numerical simulation (circles).

\section{References}

1. Mann, B.P. Sims, N.D.: Energy harvesting from the nonlinear oscillations of magnetic levitation. J. Sound Vib. 319, 515-530 (2009)

2. Daqaq, M.: Transduction of a bistable inductive generator driven by white and exponentially correlated gaussian noise. J. Sound Vib. 330, 2554-2564 (2011)

3. Quinn, D.D., Triplett, A.L., Vakakis, A.F., Bergman, L.A.: Energy harvesting from impulsive loads using intestinal essential nonlinearities. J. Vib. Acoust. 133, 011004 (2011)

4. Hamdi, M., Belhaq, M.: Quasi-periodic vibrations in a delayed van der Pol oscillator with timeperiodic delay amplitude. J. Vib. Control (2015). doi: 10.1177/1077546315597821

5. Belhaq, M., Hamdi, M.: Energy harversting from quasi-periodic vibrations. Nonlinear Dyn. DOI 10.1007/s11071-016-2668-6 (2016)

6. Nayfeh, A.H., Mook, D.T.: Nonlinear Oscillations. Wiley, New York (1979)
7. Belhaq, M., Houssni, M.: Quasi-periodic oscillations, chaos and suppression of chaos in a nonlinear oscillator driven by parametric and external excitations. Nonlinear Dyn. 18, 1-24 (1999) 